\title{
Preparation and Evaluation of Carbamazepine Sustained Release Tablets
}

\author{
Shib Sankar Saha ${ }^{1}$, Mehrina Nazmi ${ }^{2}$, Nandini Saha ${ }^{2}$ and Md. Selim Reza ${ }^{1}$ \\ ${ }^{1}$ Department of Pharmaceutical Technology, Faculty of Pharmacy, University of Dhaka, \\ Dhaka-1000, Bangladesh. \\ ${ }^{2}$ Department of Pharmacy, University of Asia Pacific, Dhanmondi, Dhaka-1209, Bangladesh
}

\begin{abstract}
The purpose of the present studies was to develop and characterize sustained release tablets of Carbamazepine, an antiepileptic drug. Tablets were prepared by direct compression method. In the formulation, the active drug was taken $200 \mathrm{mg}$ in every formulation. Methocel K4M, Methocel K15M CR, Methocel K100LV CR, Eudragit RSPO and Eudragit RLPO polymers were used as rate retarding agents in twenty four formulations (F-1 to F-24). The effect of hydrophilic polymer PEG 6000 as channeling agent with Methocel K4M was observed in four formulations (F-5 to F-8). The granules were evaluated for angle of repose, bulk density, tapped density. The tablets were subjected to average weight, diameter, thickness, weight variation, hardness, friability and in vitro dissolution studies. The granules showed satisfactory flow properties, compressibility and drug content. The in vitro dissolution study was carried using USP Apparatus-I (Rotating basket method apparatus) for 6 to 12 hours in distilled water with $1 \%$ sodium lauryl sulphate as the dissolution medium. The release mechanisms were explored and explained by Zero order, First order, Higuchi, Korsmeyer-Peppas and Hixson-Crowell equations. Primarily twelve formulations were prepared by using four variable amount of three polymers; Methocel K4M (50, 100, 150 and $200 \mathrm{mg}$ ) in the formulations from F-1 to F-4, Methocel K15M CR (50, 100, 150 and $200 \mathrm{mg})$ in the formulations from F-9 to F-12 and Methocel K100LV CR (50, 100, 150 and $200 \mathrm{mg})$ in the formulations from F-13 to F-16. Then four formulations were prepared where Methocel K4M amount was same but using variable amount of hydrophilic polymer PEG 6000 $(50,100,150$ and $200 \mathrm{mg})$. After that another eight formulations were prepared by using polymer Eudragit RSPO and Eudragit RLPO by using variable amount. In F-17 to F-20 the use of Eudragit RSPO was in variable amount (50, 100, 150 and $200 \mathrm{mg}$ ) and in F-21 to F-24 the use of Eudragit RLPO was in variable amount (50, 100, 150 and 200 $\mathrm{mg}$ ). Other excipients remained same in the formulations. Among these twenty four formulations' sustainability was studied and compared with three market product of $200 \mathrm{mg}$ tablet. It was found that effect of PEG 6000 as channeling agent subsequently decreased the sustaining action (Formulation F5-F8).
\end{abstract}

Key words: Anti epileptic, channeling agents, sustained release, direct compression.

\section{INTRDOUCTION}

Carbamazepine sustained release matrix tablets was prepared by direct compression method utilizing different grades of Hydroxylpropylmethyl cellulose (HPMC) that were Methocel K4M, Methocel K15M CR, Methocel K100LV CR and acrylic acid polymer Eudragit RSPO and Eudragit RLPO. Different grades of Methocel polymer are hydrophilic in nature and eudragit polymer are $\mathrm{pH}$ independent in nature. Oral sustained release dosage form by direct compression technique is a very simple approach of drug delivery systems which is very rational in the pharmaceutical

Correspondence to: Md. Selim Reza

Tel: 880-2-8612069; Fax: 880-2-8615583

Email: selim.du@gmail.com

Dhaka Univ. J. Pharm. Sci. 11(2): 173-180, 2012 (December) arena for its ease, compliance, faster production, avoid hydrolytic or oxidative reactions occurred during processing of dosage forms. ${ }^{1}$ Sustained or controlled drug delivery occurs while embedded within a polymer that may be natural or semisynthetic or synthetic in nature. The polymer is judiciously combined with the drug or other active ingredients in such a way that the active agent is released from the material in a predetermined fashion and released the drug at constant rate for desired time period. $^{2}$

There are number of techniques applied in the formulation and manufacturing of sustained release dosage form. However, matrix tablet prepared by direct compression method has attracted much 
attention due to its technological simplicity in comparison with other controlled release systems. Direct compression method had been applied for preparation of matrix tablet that involved simple blending of all ingredients used in the formulations and then underwent direct compression. It required fewer unit operations, less machinery, reduced number of personnel and reduced processing time, increased product stability and faster production rate. $^{3}$

There are three primary mechanisms by which active agents can be released from a delivery system: diffusion, degradation, and swelling followed by diffusion. The release of drug from the matrix tablet depends on the nature of polymer. Methocel K4M, Methocel K15M CR and Methocel K100LV CR are hydrophilic polymers that become hydrated, swollen and facilitates to diffuse the drug. ${ }^{4}$ The effect of hydrophilic polymer PEG 6000 which act as a channeling agent was evaluated on the matrix tablet with combination of Methocel K4M. Here $\mathrm{pH}$ independent swelling of Eudragit RSPO and Eudragit RLPO released the drug from the matrix. In the present study an attempt had been made to formulate carbamazepine as sustained release matrix tablet with the addition of release retarding polymers Methocel K4M, Methocel K15M CR, Methocel K100LV CR, Eudragit RSPO and Eudragit RLPO. Three market products were also evaluated.

\section{MATERIALS AND METHODS}

Drug. Carbamazepine was obtained from Beximco Pharmaceutical Ltd., Dhaka.

Polymers. Hydroxylpropyl methylcellulose Methocel K4M Premium (Colorcon USA), Hydroxypropyl methylcellulose Methocel K15M Premium (Colorcon USA), Hydroxylpropyl methylcellulose Methocel K100LV CR, Premium (Colorcon USA), Eudragit RSPO (EVONIK, Germany) Eudragit RLPO (EVONIK, Germany). ${ }^{8}$

Other excipients. Microcrystalline Cellulose (Avicel PH 102) (Hanau chemicals ltd), Poly ethylene glycol (PEG), Ludipress (BASF), Aerosil,
Talc, Magnesium Stearate were procured from local matket.

Solvents and reagents. Methanol (Merck, Germany) and Distilled water were obtained from our laboratory.

Equipments. Single punch tablet press; Simadzu UV spectrophometer, Digital $\mathrm{pH}$ meter, Electrolab tablet dissolution test machine (XXII), Sartorius electronic balance were available in the departmental laboratory.

Preparation of matrix tablet. Drug, polymer and other excipients were weighed separately for 30 tablets per formulation as per proposed formulations. The proposed formulations were coded as F-1, F-2,F3, F-4, F-5, F-6, F-7, F-8, F-9, F-10, F-11, F-12, F13, F-14, F-15, F-16, F-17, F-18, F-19, F-20, F-21, F22, F-23, F-24. The amounts of drug and excipients were expressed in milligram unit. Then active ingredient, microcrystalline cellulose, polymer and aerosil were blended for 15 minutes and then magnesium stearate, talc were added and further blended for another 1 minute. Blended mass was taken in the hopper and then die and punch were adjusted to get the desired weight of the tablet. The tablets were prepared by direct compression, the types and amounts of polymers used are shown in Table 1.

Physical evaluation of powders. The powders were evaluated for angle of repose, loose bulk density, tapped bulk density, compressibility index, total porosity etc.

Bulk density. LBD (Loose Bulk Density) and TBD (Tapped Bulk Density) were determined by $2 \mathrm{~g}$ of powder from each formula. The powder was previously lightly shaken to break any agglomerates formed and then it was placed into a $10 \mathrm{ml}$ measuring cylinder. After the initial volume was observed, the cylinder was allowed to tap under its own weight onto a hard surface from the height of $2.5 \mathrm{~cm}$ at 2 second intervals. The reading of tapping was continued until no further change in volume was noted. Using the following equation LBD and TBD was calculated: 
LBD $=$ Weight of the powder / bulk volume of the packing. TBD $=$ Weight of the powder / Tapped volume of the packing.

Compressibility index. The compressibility index of the granules was determined by Carr's compressibility index:

Carr's index $(\%)=\{(\mathrm{TBD}-\mathrm{LBD}) \mathrm{X} 100\} / \mathrm{TBD}$

Angle of repose. The angle of repose of granules was determined by the funnel method.

Total porosity. Total porosity was determined by measuring the volume occupied by a selected weighed granules were taken in a funnel. The height of the funnel was adjusted in such a way that the tip of the funnel just touched the apex of the heap of the granules. The granules were allowed to flow through the funnel freely onto the surface. The diameter of the powder cone was measured and angle of repose was calculated using the following equation. Angle of Repose $\theta=\tan ^{-1} \mathrm{~h} / \mathrm{r}$, where, $\mathrm{h}=$ Height of the powder cone, $r=$ Radius of the powder cone.

Table 1. Formulation design of Carbamazepine sustained release tablets containing Methocel K4M, Methocel K15M CR, Methocel K100LV CR, PEG 6000, Eudragit RSPO. and Eudragit RLPO.

\begin{tabular}{|c|c|c|c|c|c|c|c|c|c|c|c|c|c|}
\hline $\begin{array}{l}\text { Formu- } \\
\text { lation }\end{array}$ & $\begin{array}{c}\text { Carbama- } \\
\text { zepine } \\
\text { (mg) }\end{array}$ & $\begin{array}{l}\text { Avicell } \\
\text { PH } 102 \\
\text { (mg) }\end{array}$ & $\begin{array}{l}\text { Ludi- } \\
\text { press } \\
\text { (mg) }\end{array}$ & $\begin{array}{c}\text { Methocel } \\
\text { K4M (mg) }\end{array}$ & $\begin{array}{l}\text { PEG } \\
6000 \\
(\mathrm{mg})\end{array}$ & $\begin{array}{l}\text { Methocel } \\
\text { K15M CR } \\
\text { (mg) }\end{array}$ & $\begin{array}{l}\text { Methocel } \\
\text { K100LV } \\
\text { CR (mg) }\end{array}$ & $\begin{array}{c}\text { Eudragit } \\
\text { RSPO } \\
(\mathrm{mg})\end{array}$ & $\begin{array}{c}\text { Eudragit } \\
\text { RLPO } \\
(\mathrm{mg})\end{array}$ & $\begin{array}{c}\text { Aerosil } \\
\text { (mg) }\end{array}$ & $\begin{array}{l}\text { Magnesium } \\
\text { Stearate } \\
(\mathrm{mg})\end{array}$ & $\begin{array}{l}\text { Talc } \\
(\mathrm{mg})\end{array}$ & $\begin{array}{c}\text { Total } \\
\text { weight } \\
(\mathrm{mg})\end{array}$ \\
\hline F1 & 200 & 100 & 100 & 50 & & & & & & 2 & 1 & 1 & 454 \\
\hline F2 & 200 & 100 & 100 & 100 & & & & & & 2 & 1 & 1 & 504 \\
\hline F3 & 200 & 100 & 100 & 150 & & & & & & 2 & 1 & 1 & 554 \\
\hline F4 & 200 & 100 & 100 & 200 & & & & & & 2 & 1 & 1 & 604 \\
\hline F5 & 200 & 100 & 100 & 100 & 50 & & & & & 2 & 1 & 1 & 554 \\
\hline F6 & 200 & 100 & 100 & 100 & 100 & & & & & 2 & 1 & 1 & 604 \\
\hline F7 & 200 & 100 & 100 & 100 & 150 & & & & & 2 & 1 & 1 & 654 \\
\hline F8 & 200 & 100 & 100 & 100 & 200 & & & & & 2 & 1 & 1 & 704 \\
\hline F9 & 200 & 100 & 100 & & & 50 & & & & 2 & 1 & 1 & 454 \\
\hline F10 & 200 & 100 & 100 & & & 100 & & & & 2 & 1 & 1 & 504 \\
\hline F11 & 200 & 100 & 100 & & & 150 & & & & 2 & 1 & 1 & 554 \\
\hline $\mathrm{F} 12$ & 200 & 100 & 100 & & & 200 & & & & 2 & 1 & 1 & 604 \\
\hline F13 & 200 & 100 & 100 & & & & 50 & & & 2 & 1 & 1 & 454 \\
\hline F14 & 200 & 100 & 100 & & & & 100 & & & 2 & 1 & 1 & 504 \\
\hline F15 & 200 & 100 & 100 & & & & 150 & & & 2 & 1 & 1 & 554 \\
\hline F16 & 200 & 100 & 100 & & & & 200 & & & 2 & 1 & 1 & 604 \\
\hline F17 & 200 & 100 & 100 & & & & & 50 & & 2 & 1 & 1 & 454 \\
\hline F18 & 200 & 100 & 100 & & & & & 100 & & 2 & 1 & 1 & 504 \\
\hline F19 & 200 & 100 & 100 & & & & & 150 & & 2 & 1 & 1 & 554 \\
\hline F20 & 200 & 100 & 100 & & & & & 200 & & 2 & 1 & 1 & 604 \\
\hline $\mathrm{F} 21$ & 200 & 100 & 100 & & & & & & 50 & 2 & 1 & 1 & 454 \\
\hline $\mathrm{F} 22$ & 200 & 100 & 100 & & & & & & 100 & 2 & 1 & 1 & 504 \\
\hline F23 & 200 & 100 & 100 & & & & & & 150 & 2 & 1 & 1 & 554 \\
\hline F24 & 200 & 100 & 100 & & & & & & 200 & 2 & 1 & 1 & 604 \\
\hline
\end{tabular}

In vitro dissolution studies. Dissolution testing was performed in an "Electrolab Dissolution Tester, Germany" using Apparatus 1 (Basket method) at 100 rpm. Six tablets were evaluated per formulation in this study. The dissolution medium was $900 \mathrm{ml}$ distilled water with $1 \%$ sodium lauryl sulphate (USP method) in $37.0 \pm 0.5^{\circ} \mathrm{C}$. The amount of drug present was determined according to the USP monograph for carbamazepine tablets using UV spectrophotometer testing at $284 \mathrm{~nm} .^{6}$ In the USP monograph it is said that $65-90 \%$ drug should be released in 6-12 hours. Dissolution studies were carried over 7-12 hours in the medium.

Kinetic analysis of release data. The release of drug from sustained release dosage form was regulated by several process. These are extraction or diffusion of matrix or erosion of matrix alternatively. 
The drug dissolved in the matrix material and then released by diffusion through membrane. ${ }^{7}$ In some cases, drug may be released by osmotic process. Different kinetic equations (Zero order, First order, Higuchi release, Korsmeyer-peppas, Hixon-crowell) were applied to interpret the release rate from the tablet. The best fitted models were zero order kinetics, Korsmeyer-peppas and Hixon crowell, as these plots showed the highest linearity.

Statistical Analysis. Data from the experiments were analyzed using the Statistical Package for Social Science (SPSS) software for windows version 17 (SPSS Inc., Chicago, Illinois, USA). Statistical analysis of the results was performed by using oneway analysis of variance (ANOVA) followed by Dennett's t-test for comparisons. The limit of significance was set at $\mathrm{p}<0.05$.

Mechanism of drug release. Korsmeyer et al (1983) derived a simple relationship which described drug release from a polymeric system Equation (1). To find out the mechanism of drug release, first $60 \%$ drug release data was fitted in Korsmeyer-Peppas model:

$$
\mathrm{Mt} / \mathrm{M} \infty=\mathrm{Kt}^{\mathrm{n}} .
$$

Where, Mt / M $\infty$ is the fraction of drug released at time $\mathrm{t}, \mathrm{k}$ is the rate constant and $\mathrm{n}$ is the release exponent. The $\mathrm{n}$ value is used to characterize different release mechanisms as given in the following for cylindrical shaped matrices:

$\begin{array}{ll}\begin{array}{l}\text { Value of diffusion } \\ \text { exponent }(\mathrm{n})\end{array} & \text { Release mechanisms } \\ 0.45 & \text { Fickian diffusion } \\ 0.45<\mathrm{n}<0.89 & \text { Anomalous (non- } \\ 0.89 & \text { Fickian) diffusion } \\ \mathrm{n}>0.89 & \text { Case-II transport } \\ & \text { Super case-II transport }\end{array}$

\section{RESULTS AND DISCUSSION}

Different physical properties like angle of repose, $\mathrm{LBD}, \mathrm{TBD}$, corr's index, total porosity and in vitro dissolution studies were observed. The results are given in the following tables.

Table 2. Properties of granules of carbamazepine and excipients containing Methocel K4M, Methocell K15M CR, Methocell K100LV CR, PEG 6000, Eudragit RSPO and Eudragit RLPO.

\begin{tabular}{lcccccc}
\hline Formulations & $\begin{array}{c}\text { Angle of repose } \\
\left({ }^{\circ}\right)\end{array}$ & $\begin{array}{c}\text { Loose bulk density } \\
(\mathrm{LBD})(\mathrm{g} / \mathrm{ml})\end{array}$ & $\begin{array}{c}\text { Tapped bulk density } \\
(\mathrm{TBD})(\mathrm{g} / \mathrm{ml})\end{array}$ & $\begin{array}{c}\text { Carr's index } \\
(\%)\end{array}$ & $\begin{array}{c}\text { Hausner } \\
\text { ratio }\end{array}$ & $\begin{array}{c}\text { Total porosity } \\
(\%)\end{array}$ \\
\hline F-1 & $28.07 \pm 0.02$ & $0.418 \pm 0.01$ & $0.505 \pm 0.02$ & $17.22 \pm 0.08$ & $1.20 \pm 0.01$ & $17.14 \pm 0.01$ \\
F-2 & $26.57 \pm 0.05$ & $0.405 \pm 0.06$ & $0.5 \pm 0.04$ & $19 \pm 0.02$ & $1.23 \pm 0.03$ & $18.96 \pm 0.04$ \\
F-3 & $28.1 \pm 0.03$ & $0.461 \pm 0.08$ & $0.539 \pm 0.09$ & $14.47 \pm 0.01$ & $1.16 \pm 0.04$ & $14.29 \pm 0.03$ \\
F-4 & $23.75 \pm 0.01$ & $0.446 \pm 0.05$ & $0.539 \pm 0.09$ & $17.25 \pm 0.07$ & $1.20 \pm 0.02$ & $17.14 \pm 0.04$ \\
F-5 & $29.98 \pm 0.01$ & $0.458 \pm 0.01$ & $0.54 \pm 0.011$ & $15.18 \pm 0.02$ & $1.17 \pm 0.03$ & $15.15 \pm 0.02$ \\
F-6 & $25.17 \pm 0.03$ & $0.459 \pm 0.02$ & $0.57 \pm 0.02$ & $19.47 \pm 0.02$ & $1.24 \pm 0.01$ & $19.44 \pm 0.02$ \\
F-7 & $27.07 \pm 0.02$ & $0.471 \pm 0.04$ & $0.569 \pm 0.02$ & $17.22 \pm 0.02$ & $1.20 \pm 0.04$ & $17.24 \pm 0.02$ \\
F-8 & 26.320 .06 & $0.45 \pm 0.08$ & $0.548 \pm 0.02$ & $17.88 \pm 0.03$ & $1.21 \pm 0.02$ & $17.86 \pm 0.06$ \\
F-9 & $28.01 \pm 0.04$ & $0.469 \pm 0.04$ & $0.525 \pm 0.08$ & $10.66 \pm 0.02$ & $1.11 \pm 0.03$ & $10.71 \pm 0.05$ \\
F-10 & $32.01 \pm 0.04$ & $0.409 \pm 0.04$ & $0.478 \pm 0.07$ & $14.43 \pm 0.02$ & $1.16 \pm 0.02$ & $12.5 \pm 0.04$ \\
F-11 & $30.96 \pm 0.06$ & $0.405 \pm 0.05$ & $0.468 \pm 0.06$ & $13.46 \pm 0.01$ & $1.15 \pm 0.04$ & $9.756 \pm 0.04$ \\
F-12 & $29.73 \pm 0.02$ & $0.449 \pm 0.05$ & $0.518 \pm 0.06$ & $13.32 \pm 0.02$ & $1.15 \pm 0.03$ & $13.33 \pm 0.04$ \\
F-13 & $31.05 \pm 0.01$ & $0.423 \pm 0.05$ & $0.497 \pm 0.04$ & $14.88 \pm 0.01$ & $1.17 \pm 0.02$ & $11.11 \pm 0.02$ \\
F-14 & $28.92 \pm 0.05$ & $0.416 \pm 0.01$ & $0.493 \pm 0.03$ & $15.61 \pm 0.05$ & $1.18 \pm 0.02$ & $15.38 \pm 0.07$ \\
F-15 & 26.320 .07 & $0.409 \pm 0.01$ & $0.57 \pm 0.01$ & $28.24 \pm 0.05$ & $1.39 \pm 0.03$ & $14.29 \pm 0.07$ \\
F-16 & $28.01 \pm 0.05$ & $0.405 \pm 0.01$ & $0.569 \pm 0.03$ & $28.82 \pm 0.05$ & $1.40 \pm 0.03$ & $17.14 \pm 0.01$ \\
F-17 & $32.08 \pm 0.05$ & $0.449 \pm 0.01$ & $0.548 \pm 0.03$ & $18.06 \pm 0.08$ & $1.22 \pm 0.03$ & $15.15 \pm 0.02$ \\
F-18 & $30.96 \pm 0.07$ & $0.423 \pm 0.03$ & $0.525 \pm 0.05$ & $19.42 \pm 0.04$ & $1.24 \pm 0.04$ & $19.44 \pm 0.03$ \\
F-19 & $29.73 \pm 0.03$ & $0.416 \pm 0.04$ & $0.478 \pm 0.05$ & $12.97 \pm 0.03$ & $1.14 \pm 0.03$ & $17.24 \pm 0.04$ \\
F-20 & $31.05 \pm 0.02$ & $0.409 \pm 0.05$ & $0.46 \pm 0.08$ & $11.08 \pm 0.02$ & $1.12 \pm 0.01$ & $17.86 \pm 0.04$ \\
F-21 & $25.92 \pm 0.06$ & $0.405 \pm 0.04$ & $0.518 \pm 0.07$ & $21.81 \pm 0.02$ & $1.27 \pm 0.03$ & $10.71 \pm 0.05$ \\
F-22 & 26.320 .08 & $0.449 \pm 0.04$ & $0.497 \pm 0.06$ & $9.65 \pm 0.04$ & $1.10 \pm 0.01$ & $12.5 \pm 0.07$ \\
F-23 & $29.01 \pm 0.06$ & $0.423 \pm 0.06$ & $0.493 \pm 0.08$ & $14.19 \pm 0.05$ & $1.16 \pm 0.02$ & $9.756 \pm 0.01$ \\
F-24 & $33.01 \pm 0.06$ & $0.448 \pm 0.08$ & $0.497 \pm 0.04$ & $9.65 \pm 0.02$ & $1.37 \pm 0.03$ & $12.5 \pm 0.06$ \\
\hline & & & & & & \\
\hline
\end{tabular}


Table 3. Percentage of release of twenty four formulations and market product of Carbamazepine sustained release tablets against time.

\begin{tabular}{|c|c|c|c|c|c|c|c|c|c|c|c|c|c|c|c|c|}
\hline & Time( hr) & 0 & 0.25 & 0.5 & 1 & 2 & 3 & 4 & 5 & 6 & 7 & 8 & 9 & 10 & 11 & 12 \\
\hline \multirow{27}{*}{ 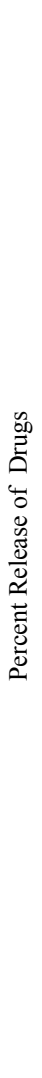 } & $\mathrm{F} 1$ & 0 & 6.35 & 8.37 & 16.00 & 32.26 & 53.90 & 70.11 & 83.52 & 97.85 & 99.09 & - & - & - & - & - \\
\hline & $\mathrm{F} 2$ & 0 & 6.26 & 8.74 & 15.26 & 30.11 & 49.88 & 64.83 & 79.30 & 84.25 & 87.30 & 93.18 & - & - & - & - \\
\hline & F3 & 0 & 5.51 & 7.62 & 11.42 & 23.45 & 43.79 & 54.03 & 61.32 & 69.98 & 77.98 & 85.79 & 96.30 & - & - & - \\
\hline & F4 & 0 & 5.89 & 8.55 & 10.79 & 19.83 & 27.30 & 35.87 & 48.07 & 59.10 & 69.78 & 80.01 & 88.79 & 94.01 & 97.79 & 98.81 \\
\hline & F5 & 0 & 6.16 & 8.93 & 15.07 & 31.23 & 50.35 & 65.22 & 80.53 & 84.57 & 88.54 & 95.65 & - & - & - & - \\
\hline & F6 & 0 & 5.98 & 9.11 & 16.09 & 33.38 & 53.73 & 67.25 & 80.72 & 86.52 & 90.61 & 97.09 & - & - & - & - \\
\hline & F7 & 0 & 6.26 & 9.58 & 16.75 & 35.35 & 54.52 & 68.50 & 83.75 & 87.26 & 91.36 & 98.12 & - & - & - & - \\
\hline & F8 & 0 & 6.72 & 10.14 & 17.51 & 35.74 & 57.05 & 69.76 & 84.10 & 87.61 & 93.76 & 98.59 & - & - & - & - \\
\hline & F9 & 0 & 4.72 & 7.52 & 13.46 & 28.20 & 50.36 & 64.77 & 73.01 & 80.80 & 87.99 & 93.39 & 98.66 & - & - & - \\
\hline & F10 & 0 & 4.61 & 7.15 & 12.24 & 27.53 & 41.78 & 52.84 & 61.88 & 72.62 & 81.95 & 87.10 & 93.79 & 97.01 & - & - \\
\hline & F11 & 0 & 4.58 & 7.14 & 11.41 & 24.92 & 38.68 & 51.84 & 62.07 & 70.49 & 77.20 & 82.86 & 88.39 & 92.48 & 98.84 & 99.12 \\
\hline & F12 & 0 & 4.68 & 6.59 & 10.84 & 23.05 & 38.27 & 48.36 & 57.07 & 64.41 & 71.61 & 75.91 & 80.99 & 84.45 & 89.79 & 94.07 \\
\hline & F13 & 0 & 6.26 & 9.02 & 16.75 & 34.13 & 56.45 & 71.57 & 84.07 & 98.49 & 99.92 & - & - & - & - & - \\
\hline & F14 & 0 & 6.72 & 9.03 & 16.75 & 32.28 & 50.85 & 66.47 & 80.31 & 86.38 & 92.61 & 97.62 & - & - & - & - \\
\hline & F15 & 0 & 5.42 & 7.43 & 12.54 & 24.95 & 44.28 & 56.76 & 64.07 & 73.51 & 81.55 & 88.65 & 99.85 & - & - & - \\
\hline & F16 & 0 & 5.79 & 8.92 & 11.53 & 21.89 & 29.66 & 37.88 & 50.10 & 61.90 & 72.70 & 82.40 & 91.59 & 93.49 & 96.89 & 98.20 \\
\hline & F17 & 0 & 1.89 & 3.86 & 6.60 & 12.44 & 15.64 & 20.46 & 26.73 & 34.92 & 39.39 & 45.67 & 52.76 & 57.50 & 62.11 & 67.69 \\
\hline & F18 & 0 & 1.98 & 4.05 & 6.23 & 11.41 & 13.58 & 18.01 & 24.25 & 28.88 & 36.16 & 42.04 & 49.28 & 56.12 & 60.90 & 65.36 \\
\hline & F19 & 0 & 1.79 & 3.77 & 5.19 & 9.35 & 12.34 & 16.38 & 21.20 & 26.82 & 32.97 & 39.56 & 45.00 & 52.36 & 57.19 & 59.19 \\
\hline & $\mathrm{F} 20$ & 0 & 1.79 & 3.58 & 4.09 & 7.48 & 10.35 & 14.27 & 19.08 & 24.58 & 30.80 & 34.20 & 40.42 & 45.78 & 51.01 & 56.10 \\
\hline & F21 & 0 & 6.07 & 8.46 & 15.62 & 30.11 & 52.20 & 66.81 & 74.98 & 85.21 & 89.10 & 95.44 & 98.49 & - & - & - \\
\hline & F22 & 0 & 6.16 & 8.28 & 14.51 & 28.15 & 51.33 & 63.98 & 73.04 & 81.76 & 85.80 & 93.04 & 96.26 & 98.57 & - & - \\
\hline & F23 & 0 & 6.26 & 7.26 & 11.43 & 25.03 & 37.67 & 47.29 & 60.27 & 70.06 & 77.69 & 86.71 & 89.49 & 94.52 & 95.88 & 97.06 \\
\hline & F24 & 0 & 5.79 & 6.51 & 9.46 & 20.81 & 34.15 & 44.85 & 56.40 & 64.29 & 70.75 & 74.48 & 77.69 & 80.92 & 84.74 & 87.29 \\
\hline & $\begin{array}{c}\text { Carbazin } \\
\mathrm{CR}(\mathrm{SK}+\mathrm{F})\end{array}$ & 0 & 3.75 & 6.39 & 10.18 & 24.43 & 38.64 & 47.53 & 62.27 & 74.38 & 82.62 & 86.48 & 89.20 & 92.37 & 95.19 & 96.54 \\
\hline & $\begin{array}{c}\text { Tegretol } \\
\text { CR(Novartis) }\end{array}$ & 0 & 8.77 & 18.91 & 39.66 & 51.35 & 62.05 & 65.60 & 70.40 & 70.88 & 75.34 & 77.44 & 80.04 & 81.23 & 85.78 & 86.84 \\
\hline & $\begin{array}{l}\text { Epilep CR } \\
\text { (Beximco) }\end{array}$ & 0 & 2.07 & 5.44 & 9.69 & 26.25 & 40.67 & 51.44 & 65.11 & 76.51 & 82.73 & 89.19 & 91.38 & 93.64 & 97.96 & 98.98 \\
\hline
\end{tabular}

Reducing the dose frequency of Carbamazepine increases patient compliance. Patients prefer to take the drug once or twice daily. Sustained release Carbamazepine prepared by the hydrophilic matrix of HPMC and pH independent polymer Eudragit RSPO, Eudragit RLPO controlled the Carbamazepine release effectively for 7-12 hour. ${ }^{8}$ Hence, these formulations can be considered as twice daily sustained release tablet of Carbamazepine. The formulation showed acceptable pharmacotechnical properties. In vitro dissolution studies indicated a steady state sustained release pattern throughout 7-12 hour of the study which was comparable to theoretical release profile. Drug release kinetics indicated that the drug release was best explained by zero order kinetics, Korsmeyer-peppas and Hixon crowell, as these plots showed the highest linearity. In the present study Twenty four formulations were formulated primarily by using four variable content of five polymers; Methocel K4M CR from formulation F1-F4 (variable content are $50 \mathrm{mg}, 100 \mathrm{mg}, 150 \mathrm{mg}, 200 \mathrm{mg}$ ), Methocel K15M CR from formulation F9-F12 (variable content are $50 \mathrm{mg}, 100 \mathrm{mg}, 150 \mathrm{mg}, 200 \mathrm{mg}$ ), Methocel K100LV CR from formulation F13-F16 (variable content are $50 \mathrm{mg}, 100 \mathrm{mg}, 150 \mathrm{mg}, 200 \mathrm{mg}$ ), Eudragit RSPO from formulation F17-F20 (variable content are $50 \mathrm{mg}, 100 \mathrm{mg}, 150 \mathrm{mg}, 200 \mathrm{mg}$ ) and Eudragit RLPO from formulation F21-F24 (variable content are $50 \mathrm{mg}, 100 \mathrm{mg}, 150 \mathrm{mg}, 200 \mathrm{mg}$ ). To see the effect of Channeling agent PEG 6000 with polymer Methocel K4M, four variable amount of PEG 6000 were prepared, F-5-F-8 (50mg, 100mg, 150mg, 200 $\mathrm{mg}$ ) where polymer Methocel K4M content remained same $(100 \mathrm{mg})$. Three market products were also studied. Only six formulations F-4, F-11, F-12, F-16, F-23 and F-24 met the release profile comparable 


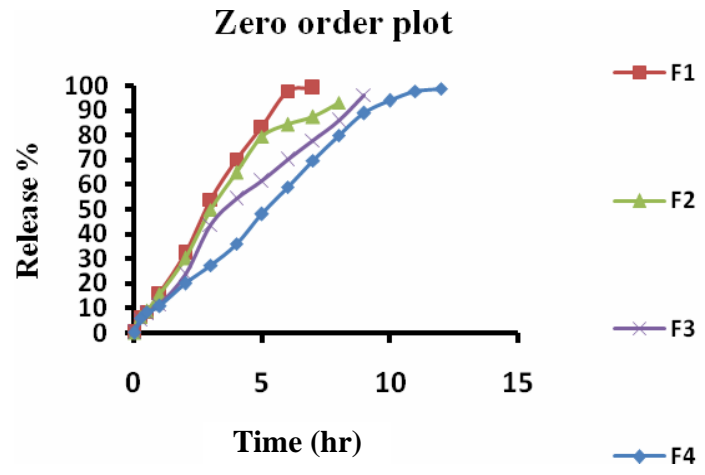

Figure 1. Zero order release kinetics of Carbamazepine sustained release tablets (F1-F4)

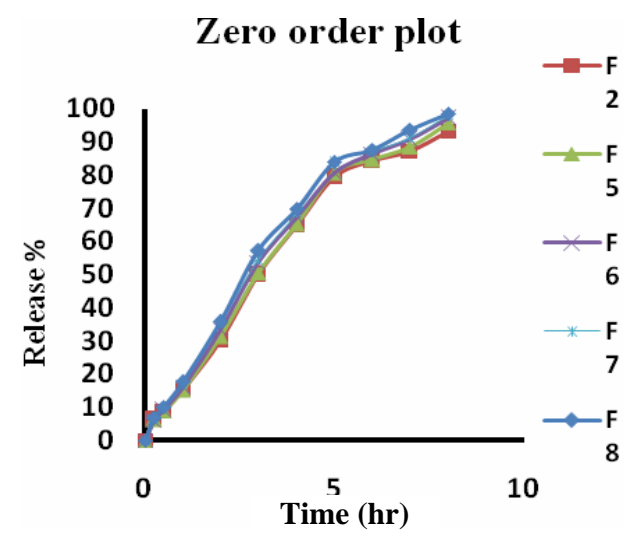

Figure 2. Zero order release kinetics of Carbamazepine sustained release tablets (F2 and F5-F8).

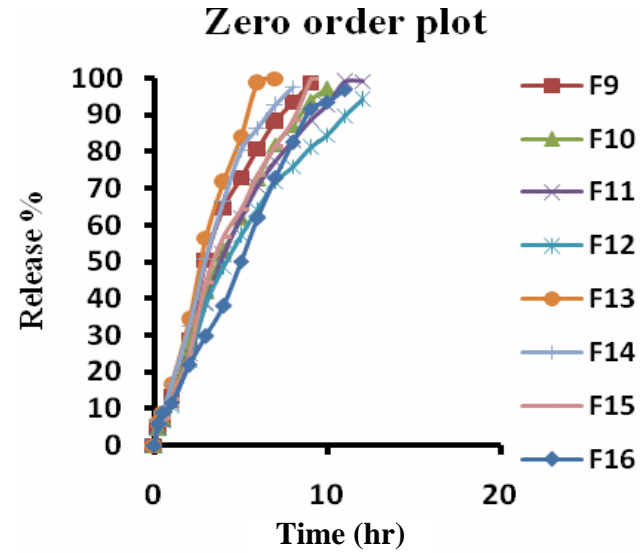

Figure 3. Zero order release kinetics of Carbamazepine sustained release tablets (F9-F16).

with market product and three formulations F-17, F18 and F-24 met the official USP release guideline of carbamazepine sustained release tablet. The drug release followed mainly super case II transport $(\mathrm{n}>0.85)$ in F-1, F-2, F-3, F-5, F-6, F-7, F-9, F-10, F-
11, F-13, F-15, F-17, F-18, F-19, F-20, anomalous or non-fickian transport $(\mathrm{n}>0.43$ and $\mathrm{n}<0.85)$ in formulations F-4, F-8, F-12, F-14, F-16, F-21, F22, F-23, F-24.

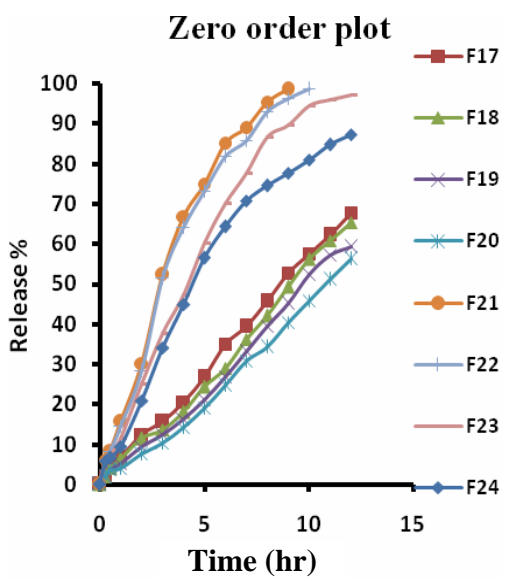

Figure 4. Zero order release kinetics of Carbamazepine sustained release tablets (F17-F24)

\section{Zero order plot}

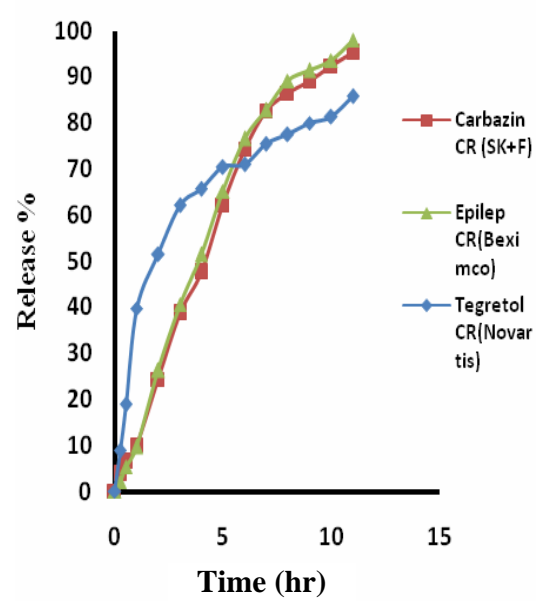

Figure 5. Zero order release kinetıcs of Market products of Carbamazepine sustained release tablets.

Impact of channeling agent (PEG 6000) on drug release from matrix tablet of Carbamazepine. Poly ethylene glycol (PEG) is a hydrophilic molecule. It is used in different formulation of poorly water soluble drugs to increase solubility. ${ }^{9}$ In formulations F-5 to F-8 variable amount of PEG 6000 was used with Methocel K4M. For formulations F-5, F-6, F-7 and F-8, the PEG 6000 content were $50 \mathrm{mg}, 100 \mathrm{mg}, 150 \mathrm{mg}$ and $200 \mathrm{mg}$, respectively. Whereas in this formulations the Methocel K4M content remained same, which was 
$100 \mathrm{mg}$. If we compare formulations F-5 to F-8 with F-2 (where methocel K4M content was same $100 \mathrm{mg}$, but here there was no use of PEG 6000) it was found that the release of drugs from the matrix tablet carbamazepine increased due to increase content of PEG 6000. It was seen that in 8 hours, from formulation F-2 (PEG 6000 was not used), the release was 93.18\%. In Formulations F-5 (50mg PEG 6000 is used) it was $95.64 \%$, in F-6 (100mg PEG 6000 is used) it was 97.08\%, in F-7 (150mg PEG 6000 is used) it was $98.12 \%$ and in F-8 (200mg PEG 6000 is used) it was $98.59 \%$ in 8 hours.

Table 4. Release rate constants and $\mathrm{R}^{2}$ values for different release kinetics of twenty four formulations and market product of Carbamazepine sustained release tablets.

\begin{tabular}{|c|c|c|c|c|c|c|c|c|c|c|c|}
\hline \multirow[t]{2}{*}{ Formulation } & \multicolumn{2}{|c|}{ Zero order } & \multicolumn{2}{|c|}{ First order } & \multicolumn{2}{|c|}{ Higuchi } & \multicolumn{3}{|c|}{ Korsmeyer-Peppas } & \multicolumn{2}{|c|}{ Hixson- Crowell } \\
\hline & $\mathrm{K}_{0}$ & $\mathrm{R}^{2}$ & $\mathrm{~K}_{1}$ & $\mathrm{R}^{2}$ & $\mathrm{~K}_{\mathrm{h}}$ & $\mathrm{R}^{2}$ & $\mathrm{n}$ & $\mathrm{K}_{\mathrm{Km}}$ & $\mathrm{R}^{2}$ & $\mathrm{~K}_{\mathrm{HC}}$ & $\mathrm{R}^{2}$ \\
\hline F1 & 15.31 & 0.983 & -0.617 & 0.869 & 42.64 & 0.95 & 0.906 & 0.184 & 0.987 & 0.663 & 0.958 \\
\hline $\mathrm{F} 2$ & 12.44 & 0.961 & 0.327 & 0.982 & 37.77 & 0.963 & 0.852 & 0.177 & 0.987 & 0.442 & 0.992 \\
\hline F3 & 10.71 & 0.984 & -0.29 & 0.881 & 34.13 & 0.963 & 0.856 & 0.148 & 0.984 & 0.382 & 0.965 \\
\hline F4 & 9.258 & 0.993 & -0.287 & 0.876 & 32.06 & 0.937 & 0.796 & 0.137 & 0.976 & 0.349 & 0.959 \\
\hline F5 & 12.66 & 0.964 & -0.359 & 0.963 & 38.39 & 0.964 & 0.858 & 0.178 & 0.988 & 0.467 & 0.991 \\
\hline F6 & 12.84 & 0.961 & -0.393 & 0.952 & 39.08 & 0.968 & 0.864 & 0.182 & 0.99 & 0.494 & 0.993 \\
\hline F7 & 12.95 & 0.957 & -0.428 & 0.931 & 39.51 & 0.969 & 0.852 & 0.19 & 0.99 & 0.515 & 0.989 \\
\hline F8 & 13.03 & 0.955 & -0.46 & 0.927 & 39.84 & 0.97 & 0.834 & 0.198 & 0.989 & 0.535 & 0.991 \\
\hline F9 & 11.6 & 0.96 & -0.393 & 0.902 & 37.55 & 0.969 & 0.907 & 0.155 & 0.988 & 0.465 & 0.986 \\
\hline F10 & 10.2 & 0.977 & -0.31 & 0.934 & 34.64 & 0.973 & 0.878 & 0.143 & 0.993 & 0.391 & 0.989 \\
\hline F11 & 9.273 & 0.969 & -0.306 & 0.865 & 33.28 & 0.976 & 0.862 & 0.138 & 0.991 & 0.366 & 0.977 \\
\hline F12 & 8.029 & 0.96 & -0.209 & 0.972 & 30.37 & 0.98 & 0.834 & 0.133 & 0.988 & 0.285 & 0.996 \\
\hline F13 & 15.38 & 0.981 & -0.81 & 0.782 & 43.01 & 0.956 & 0.902 & 0.19 & 0.991 & 0.722 & 0.947 \\
\hline F14 & 12.93 & 0.969 & -0.416 & 0.94 & 39.16 & 0.967 & 0.842 & 0.187 & 0.989 & 0.509 & 0.991 \\
\hline F15 & 11.14 & 0.984 & -0.453 & 0.648 & 35.54 & 0.964 & 0.869 & 0.151 & 0.988 & 0.459 & 0.902 \\
\hline F16 & 9.291 & 0.989 & -0.283 & 0.909 & 32.49 & 0.953 & 0.79 & 0.143 & 0.985 & 0.359 & 0.974 \\
\hline F17 & 5.665 & 0.997 & -0.088 & 0.975 & 20.47 & 0.93 & 0.916 & 0.0662 & 0.995 & 0.148 & 0.991 \\
\hline F18 & 5.451 & 0.992 & -0.083 & 0.96 & 19.5 & 0.906 & 0.891 & 0.0639 & 0.987 & 0.141 & 0.975 \\
\hline F19 & 5.064 & 0.991 & -0.074 & 0.965 & 18.07 & 0.901 & 0.902 & 0.057 & 0.984 & 0.126 & 0.976 \\
\hline F20 & 4.617 & 0.991 & -0.064 & 0.964 & 16.42 & 0.894 & 0.896 & 0.051 & 0.972 & 0.112 & 0.976 \\
\hline F21 & 11.65 & 0.953 & -0.409 & 0.935 & 37.87 & 0.97 & 0.848 & 0.176 & 0.987 & 0.479 & 0.993 \\
\hline F22 & 10.48 & 0.942 & -0.379 & 0.946 & 36.24 & 0.972 & 0.829 & 0.171 & 0.982 & 0.439 & 0.996 \\
\hline F23 & 8.916 & 0.954 & -0.29 & 0.965 & 32.87 & 0.973 & 0.805 & 0.149 & 0.982 & 0.353 & 0.994 \\
\hline F24 & 7.845 & 0.945 & -0.172 & 0.996 & 29.03 & 0.973 & 0.804 & 0.133 & 0.974 & 0.251 & 0.991 \\
\hline $\begin{array}{l}\text { Carbazin CR } \\
(\mathrm{SK}+\mathrm{F})\end{array}$ & 9.406 & 0.955 & -0.278 & 0.982 & 33.14 & 0.956 & 0.901 & 0.126 & 0.986 & 0.34 & 0.991 \\
\hline $\begin{array}{l}\text { Tegretol CR } \\
\text { (Novartis) }\end{array}$ & 6.639 & 0.787 & -0.156 & 0.938 & 25.13 & 0.94 & 0.553 & 0.278 & 0.909 & 0.215 & 0.901 \\
\hline $\begin{array}{l}\text { Epilep CR } \\
\text { (Beximco) }\end{array}$ & 9.665 & 0.951 & -0.313 & 0.954 & 34.62 & 0.96 & 1.03 & 0.106 & 0.983 & 0.386 & 0.995 \\
\hline
\end{tabular}

\section{CONCLUSION}

The experiment revealed that Methocel K4M, Methocel K15M CR, Methocel K100LV CR, Eudragit RSPO and Eudragit RLPO in varying proportions controlled the carbamazepine release effectively for 7-12 hours. The effect of wetting agent PEG 6000 was also evaluated with Methocel K4M. ${ }^{10}$ In most of the cases, the release kinetics of carbamazepine depended on the content of polymers in the tablets. It was seen that from the above formulations only six formulations were comparable with market products and three formulations met the USP guidelines. The use of channeling agent PEG 6000 decreased the sustaining action of the tablets.

\section{ACKNOWLEDGEMENT}

The author like to thank Beximco Pharmaceuticals limited for providing Carbamazepine. 


\section{REFERENCES}

1. Longer, M.A., Robinson and J.R. 1990. Sustained Release Drug Delivery System of Remington's Pharmaceutical Science, Chapter $91,18^{\text {th }}$ ed. pp. 1676-1690.

2. Lordi, N.G. 1990. Sustained Release Dosage Forms (Lachman, L., Lieberman, H.A. and Kanig, J. L. Eds.), Varghese Publishing House, Bombay, pp. 430-456.

3. R.F. Shangraw and D.A. Demarest Jr. 1993. A survey of current industrial practices in the formulation and manufacture of tablets and capsules. Pharm. Technol. 17, 32.

4. Bidah D. and Vernaud J.M. 1991. Dosage forms with a polymer matrix and swelling polymer. Intl. J. Pharmaceutics. 77, 81-87.

5. Arthur H. Kibbe 2000. Handbook of Pharmaceutical Excipients. American Pharmaceutical Association, Washington D.C. $3^{\text {rd }}$ ed. p. 129.
6. United States Pharmacopoeia 29th Edition, NF 24. 2006

7. D. Renoylds 1998. Polymer erosion and drug-release characteristics of hydroxypropyl methylcellulose matrices. $J$. Pharm. Sci. 87, 1115-1123.

8. Wadher, Kakde and Umekar J. 2011. Development of a sustained-release tablet of metformin hydrochloride containing hydrophilic eudragit and ethyl cellulose polymer. Pharmacie Globale Intl. J. Comprehen. Pharm. 2(5).

9. Buchi N. Nalluri, S. Vidyasagar and K. M. Maheswari. 2012. Effect of excipients on Oxcarbazepine release from modified release matrix tablets. J. Appl. Pharm. Sci. 2, 150158. 